Comparing long monthly Chinese and selected European temperature series using the Vector Seasonal Shifting Mean and Covariance Autoregressive model

Changli He, Jian Kang, Timo Teräsvirta and Shuhua Zhang

CREATES Research Paper 2019-19 


\title{
Comparing long monthly Chinese and selected European temperature series using the Vector Seasonal Shifting Mean and Covariance Autoregressive model
}

\author{
Changli He*, Jian Kang*», Timo Teräsvirta ${ }^{\dagger \ddagger}$ and Shuhua Zhang* \\ *Coordinated Innovation Center for Computable \\ Modeling in Management Science, Tianjin University \\ of Finance and Economics \\ $\diamond$ School of Accounting and Finance, The Hong Kong \\ Polytechnic University \\ ${ }^{\dagger}$ CREATES, Aarhus University \\ ${ }_{\ddagger}^{\ddagger}$ C.A.S.E., Humboldt-Universität zu Berlin
}

October 24, 2019

\begin{abstract}
The purpose of this paper is to study differences in long monthly Asian and European temperature series. The longest available Asian series are those of Beijing and Shanghai, and they are compared with the ones for St Petersburg, Dublin and Uccle that have a rather different climate. The comparison is carried out in the Vector Shifting Mean and Covariance Autoregressive model that the authors have previously used to analysed 20 long European temperatute series. This model gives information about mean shifts in these five temperature series as well as (error) correlations between them. The results suggest, among other things, that warming has begun later in China than in Europe, but that the change in the summer months in both Beijing and Shanghai has been quite rapid.

Keywords. Climate change, changing seasonality; long monthly Chinese temperature series; nonlinear model; nonlinear time series; time-varying correlation, time-varying variance, time-varying vector smooth transition autoregression
\end{abstract}


JEL Classification Codes: C32; C52; Q54

Acknowledgements. A large part of this research was carried out when the third author was visiting the Tianjin University of Finance and Economics. Kind hospitality of this institution is gratefully acknowledged. The second author acknowledges support from the Tianjin Natural Science Foundation, Grant No. 17JCYBJC43300. We wish to thank Glen Wade for making his code for joint estimation of error variances and correlations available to us. Any errors and shortcomings in this work remain our responsibility.

\section{Introduction}

The purpose of the paper is to compare monthly average changes in temperature over a long period and to contrast Asian locations with selected European ones. This is important as one would like to obtain as much information as possible of long-run effects of the climate change on temperatures by season in various locations in the world. The two Asian cities, Beijing and Shanghai, are selected because they possess the longest monthly temperature records in China and Asia. To the best of our knowledge, long monthly Asian temperature time series have not been studied in detail before. The set of long series studied by Hillebrand and Proietti (2017) contained 16 European and two North American series but no Asian ones. They estimated a separate structural time series model for each series.

In previous work, He, Kang, Teräsvirta and Zhang (2019a) studied shifts in average temperatures and correlations between them using 20 European locations extending from Trondheim in the north to Milan in the south. This was done using the Vector Seasonal Shifting Mean and Covariance Autoregressive (VSSMC-AR) model developed for the purpose. The model can take into account time-variation both in the mean, error and correlation parameters of the model. The time-varying parameters contain interpretable information about seasonal long-run temperature changes as well as relationships between seasonal changes in different locations. Among other things, the VSSMC-AR model allows estimating (error) correlations between Asian and European locations or stations and thus drawing conclusions on relationships between them.

In this work, we consider three European locations that were not included in He et al. (2019a) or Hillebrand and Proietti (2017): Dublin, Uccle and St Petersburg. St Petersburg is chosen to represent Northern Europe. It is selected, among other things, because winters there can be cold as they are in Beijing. The other locations are Dublin and Uccle, of which the latter is 
the site of the Royal Meteorological Institute of Belgium. They are selected because they, Dublin in particular, lie close to the Atlantic Ocean and are in that sense quite different from St Petersburg. This is why comparing them with the more continental St Petersburg and the two Chinese cities can be interesting. We hope to gain information not only about seasonal correlations between European and Chinese series but also about these correlations between Dublin and Uccle, clearly affected by the Atlantic weather systems, on the one hand and St Petersburg, further away from the Atlantic Ocean, on the other. Besides, the results can be compared to ones reported by $\mathrm{He}$ et al. (2019a).

The plan of the paper is as follows. The VSSMC-AR model is presented in Section 2. Section 3 contains information about the five time series. Model building is described in Section 4 and the empirical results discussed in Section 5. Final remarks appear in Section 6. Figures can be found in the Appendix A and additional tables in Appendix B.

\section{The model}

The VSSMC-AR model developed by He et al. (2019a) is a multivariate generalisation of the Shifting Mean Autoregressive model by González and Teräsvirta (2008) and its seasonal version introduced in He, Kang, Teräsvirta and Zhang (2019b). In the following the main features of the model are recapitulated. The mean component of the $N$-dimensional VSSMC-AR model for unit or season $s$ is defined as follows:

$$
\mathbf{y}_{S k+s}=\sum_{j=1}^{S} \boldsymbol{\delta}_{j}\left(\frac{S k+j}{S K}\right) D_{S k+s}^{(j)}+\sum_{i=1}^{p} \boldsymbol{\Phi}_{i} \mathbf{y}_{S k+s-i}+\boldsymbol{\varepsilon}_{S k+s}
$$

$k=0,1, \ldots, K-1$, where $\mathbf{y}_{S k+s}=\left(y_{1, S k+s}, \ldots, y_{N, S k+s}\right)^{\prime}$ contains the observations, $D_{S k+s}^{(j)}=1$ when $j=s$, zero otherwise $\left(D_{S k+s}^{(j)}\right.$ is the $j$ th seasonal dummy variable), $\boldsymbol{\varepsilon}_{S k+s}$ is an $N \times 1$ error vector with mean zero, and the roots of $\left|\mathbf{I}-\sum_{i=1}^{p} \boldsymbol{\Phi}_{i} z^{i}\right|=0$ lie outside the unit circle. Furthermore, $S$ is the number of seasons, and $K$ is the total number of seasonal cycles. The vector $\boldsymbol{\delta}_{j}\left(\frac{S k+j}{S K}\right)=\left(\delta_{1 j}\left(\frac{S k+j}{S K}\right), \ldots, \delta_{N j}\left(\frac{S k+j}{S K}\right)\right)^{\prime}$ is the vector of seasonal dummy variables for season $j$. The $j$ th time-varying coefficient $\delta_{n j}\left(\frac{S k+j}{S K}\right)$ of equation $n$ equals

$$
\delta_{n j}\left(\frac{S k+j}{S K}\right)=\delta_{n j 0}+\sum_{i=1}^{q_{n j}} \delta_{n j i} g_{n j i}\left(\frac{S k+j}{S K} ; \gamma_{n j i}, \mathbf{c}_{n j i}\right)
$$


where the transition variable

$$
g_{n j i}\left(\frac{S k+j}{S K} ; \gamma_{n j i}, c_{n j i}\right)=\left(1+\exp \left\{-\gamma_{n j i}\left(\frac{S k+j}{S K}-c_{n j i}\right)\right\}\right)^{-1}
$$

or

$$
g_{n j i}\left(\frac{S k+j}{S K} ; \gamma_{n j i}, c_{1 n j i}, c_{2 n j i}\right)=1-\exp \left\{-\gamma_{n j i}\left(\frac{S k+j}{S K}-c_{n j i}\right)^{2}\right\}
$$

where $\gamma_{n j i}>0$ both in (3) and in (4). At time $S k+s$, the mean component (1) may be written as

$$
\mathbf{y}_{S k+s}=\boldsymbol{\delta}_{s}\left(\frac{S k+s}{S K}\right)+\sum_{i=1}^{p} \boldsymbol{\Phi}_{i} \mathbf{y}_{S k+s-i}+\boldsymbol{\varepsilon}_{S k+s} .
$$

Assume, for notational simplicity, that the transitions in (2) are of type (3), that is, described by logistic functions. To fix notation, let $\boldsymbol{\theta}_{n s}=$ $\left(\boldsymbol{\delta}_{n s}^{\prime}, \boldsymbol{\gamma}_{n s}^{\prime}, \mathbf{c}_{n s}^{\prime}\right)^{\prime}$, where $\boldsymbol{\delta}_{n s}=\left(\delta_{n s 0}, \delta_{n s 1}, \ldots, \delta_{n s r_{s}}\right)^{\prime}$ is an $\left(r_{n s}+1\right) \times 1$ vector, whereas $\gamma_{n s}=\left(\gamma_{n s 1}, \ldots, \gamma_{n s r_{s}}\right)^{\prime}$ and $\mathbf{c}_{n s}=\left(c_{n s 1}, \ldots, c_{n s r_{s}}\right)^{\prime}$ for $n=1, \ldots, N$ are $r_{n s} \times 1$ vectors. Let $\boldsymbol{\theta}_{s}=\left(\boldsymbol{\theta}_{1 s}^{\prime}, \ldots, \boldsymbol{\theta}_{N s}^{\prime}\right)^{\prime}$. The $N p \times N$ parameter matrix of the autoregressive component equals $\boldsymbol{\Phi}=\left(\boldsymbol{\Phi}_{1}, \ldots, \boldsymbol{\Phi}_{p}\right)$, where $\boldsymbol{\Phi}_{i}=\left[\phi_{i m n}\right]$, $m, n=1, \ldots, N, i=1, \ldots, p$. In the application of this paper, $\boldsymbol{\Phi}_{i}, i=1, \ldots, p$, are diagonal matrices, because feedback from one location to the other can be excluded.

The error process $\left\{\boldsymbol{\varepsilon}_{S k+s}\right\}$ is assumed to have a seasonally time-varying covariance matrix. The error vector is decomposed similarly to multivariate ARCH or GARCH models as

$$
\varepsilon_{S k+s}=\mathbf{V}_{S k+s} \mathbf{z}_{S k+s}
$$

where $\mathbf{z}_{S k+s} \sim \operatorname{iid} \mathcal{N}\left(\mathbf{0}, \mathbf{P}_{S k+s}\right)$ and $\mathbf{P}_{S k+s}$ is a seasonally time-varying correlation matrix. It follows that the covariance matrix $\mathbf{H}_{S k+s}=\mathrm{E} \varepsilon_{S k+s} \varepsilon_{S k+s}^{\prime}=$ $\mathbf{V}_{S k+s} \mathbf{P}_{S k+s} \mathbf{V}_{S k+s}$. The deterministic diagonal matrix $\mathbf{V}_{S k+s}=\operatorname{diag}\left(\sigma_{1 s}\left(\frac{S k+s}{S K}\right)\right.$, $\left.\ldots, \sigma_{N s}\left(\frac{S k+s}{S K}\right)\right)$ contains the time-varying error standard deviations for season $s$. The error variance of the $n$th equation at season $s$ equals

$$
\sigma_{n s}^{2}\left(\frac{S k+s}{S K}\right)=\sum_{j=1}^{S} \sigma_{n j}^{2}\left(\frac{S k+j}{S K}\right) D_{S k+s}^{(j)}
$$

where

$$
\sigma_{n j}^{2}\left(\frac{S k+j}{S K}\right)=\sigma_{n j 0}^{2}+\sum_{i=1}^{r_{n j}} \omega_{n j i} g_{n j i}^{(v)}\left(\frac{S k+j}{S K} ; \gamma_{n j i}^{(v)}, c_{n j i}^{(v)}\right)
$$


Similarly to (2), the $j$ th transition function in equation $n$ equals either

$$
g_{n j i}^{(v)}\left(\frac{S k+j}{S K} ; \gamma_{n j i}^{(v)}, c_{n j i}^{(v)}\right)=\left(1+\exp \left\{-\gamma_{n j i}^{(v)}\left(\frac{S k+j}{S K}-c_{n j i}^{(v)}\right)\right\}\right)^{-1}
$$

or

$$
g_{n j i}^{(v)}\left(\frac{S k+j}{S K} ; \gamma_{n j i}^{(v)}, c_{n j i}^{(v)}\right)=1-\exp \left\{-\gamma_{n j i}^{(v)}\left(\frac{S k+j}{S K}-c_{n j i}^{(v)}\right)^{2}\right\} .
$$

In (9) and (10), $\gamma_{n j i}^{(v)}>0, i=1, \ldots, r_{n j} ; j=1, \ldots, S$ and $n=1, \ldots, N$. To guarantee positivity of each element in (8), $\sigma_{n j 0}^{2}>0$ and $\sigma_{n j 0}^{2}+\sum_{i=1}^{q} \omega_{n j i}>0$ for $q=1, \ldots, r_{n j}, n=1, \ldots, N$, and $j=1, \ldots, S$. This definition imposes restrictions on $\omega_{n j i}, i=1, \ldots, r_{n j}$. For the individual season $s$ it conforms to the one in Silvennoinen and Teräsvirta (2016). To fix notation, let $\boldsymbol{\theta}_{s}^{(v)}=$ $\left(\boldsymbol{\theta}_{1 s}^{(v) \prime}, \ldots, \boldsymbol{\theta}_{N s}^{(v) \prime}\right)^{\prime}$ be the vector of parameters of season $s$ in the $N$ equations, where $\boldsymbol{\theta}_{n s}^{(v)}=\left(\sigma_{n s 0}^{2}, \boldsymbol{\omega}_{n s}^{\prime}, \boldsymbol{\gamma}_{n s}^{(v) \prime}, \mathbf{c}_{n s}^{(v) \prime}\right)^{\prime}$ with $\boldsymbol{\omega}_{n s}=\left(\omega_{n s 1}, \ldots, \omega_{n s r_{s}}\right)^{\prime}, \boldsymbol{\gamma}_{n s}^{(v)}=$ $\left(\gamma_{n s 1}^{(v)}, \ldots, \gamma_{n s r_{s}}^{(v)}\right)^{\prime}$, and $\mathbf{c}_{n s}^{(v)}=\left(c_{1 n s 1}^{(v)}, \ldots, c_{1 n s r_{s}}^{(v)}\right)^{\prime}$, for $n=1, \ldots, N$.

The error correlation matrix for season $s$ has the following form:

$$
\mathbf{P}_{S k+s}=\sum_{j=1}^{S}\left\{\left(1-g_{j}^{(c)}\left(\frac{S k+j}{S K}\right)\right) \mathbf{P}_{(j 1)}+g_{j}^{(c)}\left(\frac{S k+j}{S K}\right) \mathbf{P}_{(j 2)}\right\} D_{S k+s}^{(j)}
$$

where $\mathbf{P}_{(j 1)}$ and $\mathbf{P}_{(j 2)}$ are $N \times N$ positive definite correlation matrices, $j=$ $1, \ldots, S$. Furthermore, in (11),

$$
g_{j}^{(c)}\left(\frac{S k+j}{S K} ; \gamma_{j}^{(c)}, c_{j}^{(c)}\right)=\left(1+\exp \left\{-\gamma_{j}^{(c)}\left(\frac{S k+j}{S K}-c_{j}^{(c)}\right)\right\}\right)^{-1}
$$

or

$$
g_{j}^{(c)}\left(\frac{S k+j}{S K} ; \gamma_{j}^{(c)}, c_{j}^{(c)}\right)=1-\exp \left\{-\gamma_{j}^{(c)}\left(\frac{S k+j}{S K}-c_{j}^{(c)}\right)^{2}\right\}
$$

where $\gamma_{j}^{(c)}>0$ for $j=1, \ldots, S$. Since both (12) and (13) are bounded between zero and one, as a convex combination of two positive definite correlation matrices $\mathbf{P}_{S k+s}$ is positive definite for all $S k+s$; see, for example, Berben and Jansen (2005) or Silvennoinen and Teräsvirta (2005, 2015).

\section{Data}

Information about the weather stations can be found in Table 1 and their locations can be seen in Figure 1. The time series are monthly and end in 2018. They have been obtained from the KNMI Climate Explorer website (http://climexp.knmi.nl/start.cgi). The St Petersburg series is about as long as the series considered in He et al. (2019a) and hence much longer 


\begin{tabular}{lllll}
\hline Station & & & & \\
& Latitude & Longitude & Elevation, $m$ & Period \\
\hline Beijing & $39.93 \mathrm{~N}$ & $116.28 \mathrm{E}$ & 55 & $1841-2018$ \\
Dublin & $53.43 \mathrm{~N}$ & $-6.25 \mathrm{E}$ & 85 & $1831-2018$ \\
Shanghai & $31.20 \mathrm{~N}$ & $121.40 \mathrm{E}$ & 7 & $1847-2018$ \\
St Petersburg & $59.97 \mathrm{~N}$ & $30.30 \mathrm{E}$ & 6 & $1752-2018$ \\
Uccle & $50.48 \mathrm{~N}$ & $4.20 \mathrm{E}$ & 104 & $1833-2018$ \\
\hline
\end{tabular}

Table 1: Location of stations and time span for the four long monthly average temperature series

than the other four. All five stations, Shanghai and St Petersburg in particular, have a rather low elevation. The two Chinese cities lie more south than the European ones, but the Gulf Stream to some extent compensates the differences in latitude. Despite its low latitude compared to St Petersburg, Dublin and Uccle, the mean temperatures in Beijing from November to February are substantially lower than in Dublin and Uccle. They are, however, not as low as those of St Petersburg.

The series contain occasional missing values. The longest stretches appear in the Beijing series, comprising the years 1856 to 1858 and 1862 to 1868 . They have been approximated using the Kalman Filter. The correlations are estimated for the period 1847-2018, determined by the shortest (Shanghai) series. More of this in Section 5.3.

\section{Modelling}

\subsection{The seasonal mean component}

From (1) and (2) it is seen that the VSSMC-AR model is not identified if at least one season $s$ for any equation $n$ has a constant coefficient. This renders the standard specification tests useless. Testing is carried out one equation and one season at a time. For equation $n$ and season $s$, the null hypothesis is chosen as $\gamma_{n s}=0$ in (2). In order to circumvent the identification problem and carry out the test we approximate the alternative as suggested by Saikkonen and Luukkonen (1988) and Luukkonen, Saikkonen and Teräsvirta (1988). This involves approximating the coefficient defined either by (3) or (4) by a polynomial developed around the null hypothesis $\gamma_{n s}=0$. Thus, 
using a third-order polynomial as an example, the approximation becomes

$$
\begin{aligned}
y_{n, S k+s}= & \widetilde{\delta}_{n s 0}+\widetilde{\delta}_{n s 1}\left(\frac{S k+s}{S K}\right)+\widetilde{\delta}_{n s 2}\left(\frac{S k+s}{S K}\right)^{2}+\widetilde{\delta}_{n s 3}\left(\frac{S k+s}{S K}\right)^{3} \\
& +\sum_{i=1}^{p} \phi_{n s i} y_{n, S k+s-i}+\varepsilon_{S k+s}^{*}
\end{aligned}
$$

where $\varepsilon_{S k+s}^{*}=\varepsilon_{S k+s}+R_{3}((S k+s) / S K)$, with $R_{3}((S k+s) / S K)$ being the remainder. For $j \neq s$, the equation is assumed to have the form

$$
y_{n, S k+j}=\delta_{n j 0}+\sum_{i=1}^{p} \phi_{n j i} y_{n, S k+j-i}+\varepsilon_{S k+j}^{*}
$$

for $k=0,1, \ldots, K-1$, that is, stability of each seasonal coefficient in equation $n$ is tested separately, keeping the other $S-1$ coefficients constant also under the alternative. The null hypothesis $\mathrm{H}_{03}^{\prime}: \widetilde{\delta}_{n s 1}=\widetilde{\delta}_{n s 2}=\widetilde{\delta}_{n s 3}=0$ in (14). Under this hypothesis, $R_{3}((S k+s) / S K)=0$, which implies $\varepsilon_{S k+s}^{*}=$ $\varepsilon_{S k+s}$. When one constructs a Lagrange multiplier type for testing $\mathrm{H}_{03}^{\prime}$, the remainder therefore does not affect the asymptotic theory.

As Lin and Teräsvirta (1994) pointed out, the standard asymptotic theory applies even when the regressors are deterministic polynomials as in (14). Thus the usual $\chi^{2}$-statistic has an asymptotic null distribution with three degrees of freedom. There is one caveat, however, in that the errors of the VSSMC-AR model are heteroskedastic. The test statistic has to be robustified against this complication, and this is done following Wooldridge (1990).

The choice between (3) and (4) is decided using the heuristic rule in Teräsvirta (1994), based on the definition of $\widetilde{\delta}_{n s 1}, \widetilde{\delta}_{n s 2}$ and $\widetilde{\delta}_{n s 3}$ as functions of the parameters in (3) and (4). If the null hypothesis $\mathrm{H}_{03}^{\prime}$ is rejected, test $\mathrm{H}_{02}^{\prime}: \widetilde{\delta}_{n s 1}=\widetilde{\delta}_{n s 2}=0$ given $\widetilde{\delta}_{n s 3}=0$ and $\mathrm{H}_{01}^{\prime}: \widetilde{\delta}_{n s 1}=0$ given $\widetilde{\delta}_{n s 2}=\widetilde{\delta}_{n s 3}=0$. If the rejection of $\mathrm{H}_{02}^{\prime}$, measured by the $p$-value, is strongest of the three, select the exponential function (4), otherwise settle for the logistic function (3).

If the null hypothesis is rejected for $r_{n} \leq S$ seasons for equation $n, n=$ $1, \ldots, N$, the corresponding mean equation with $r_{n}$ time-varying coefficients is estimated equation by equation. It is then tested (equation by equation) against the alternative with another transition for these $r_{n}$ seasons. It turns out that in the present application, one transition is enough for all $S$ seasons (12 months) and the five equations. 


\subsection{The seasonal variance component}

The error variances of the VSSMC-AR model are time-varying, but as is obvious from (8), the model is not identified if at least one of them is constant over time. For this reason, constancy has to be tested before the (timevarying) variances are estimated and constancy of correlations tested. In the present application there is seasonal variation in the errors, and because of this, constancy over time is tested separately for each season. Testing is carried out using the polynomial approximation to the alternative as in $\mathrm{He}$ et al. (2019a). This implies approximating the error variance for equation $n$ and season $j$. As in Section 4.1, taking a third-order polynomial as an example, the approximation looks as follows:

$$
\begin{aligned}
\sigma_{n j}^{2}\left(\frac{S k+j}{S K}\right)= & \widetilde{\sigma}_{n j 0}^{2}+\widetilde{\omega}_{n j 1} \frac{S k+j}{S K}+\widetilde{\omega}_{n j 2}\left(\frac{S k+j}{S K}\right)^{2} \\
& +\widetilde{\omega}_{n j 3}\left(\frac{S k+j}{S K}\right)^{3}+R_{3}\left(\frac{S k+j}{S K} ; \gamma_{n j i}^{(v)}, c_{n j i}^{(v)}\right)
\end{aligned}
$$

where $R_{3}\left(\frac{S k+j}{S K} ; \gamma_{n j i}^{(v)}, c_{n j i}^{(v)}\right)$ is the remainder. The null hypothesis is $\mathrm{H}_{0}: \widetilde{\omega}_{n j 1}=$ $\widetilde{\omega}_{n j 2}=\widetilde{\omega}_{n j 3}=0$ in (15). Since the remainder equals zero under the null hypothesis even here, it does not affect asymptotic theory when the test is constructed using the Lagrange multiplier (LM) principle. Thus, when the null hypothesis holds, the LM-statistic has an asymptotic $\chi^{2}$-distribution with three degrees of freedom.

\subsection{The seasonal correlations}

It is seen from (11) that there is an identification problem also in the correlation matrix. When the correlations are constant for season $s, \mathbf{P}_{(s 1)}=\mathbf{P}_{(s 2)}$, and the parameters in the transition function (12) or (13) are unidentified nuisance parameters. Alternatively, $\gamma_{s}^{(c)}=0$ makes the transition function constant, in which case the location parameter $c_{j}^{(c)}$ is a nuisance paramete, and, besides, there is not enough information in the data to estimate both $\mathbf{P}_{(s 1)}$ and $\mathbf{P}_{(s 2)}$ consistently. Stability of the correlation matrix has therefore to be tested first and the time-varying correlation matrix estimated only if the null hypothesis is rejected. For details, see He et al. (2019a). 


\section{Results}

\subsection{Shifting seasonal means}

\subsubsection{Definition of the shifting seasonal mean}

In order to define the shifting seasonal mean, let the equation $n$ of the VSSMC-AR model (5) be

$$
y_{n, S k+s}=\delta_{n s}\left(\frac{S k+s}{S K}\right)+\sum_{i=1}^{p} \phi_{n i} y_{n, S k+s-i}+\varepsilon_{n, S k+s} .
$$

It follows that the corresponding shifting mean at $S k+s$ equals

$$
\mathrm{E} y_{n, S k+s}=\left(1-\sum_{i=1}^{p} \phi_{n i}\right)^{-1} \delta_{n s}\left(\frac{S k+s}{S K}\right)+O\left(\frac{1}{S K}\right)
$$

where $O\left(\frac{1}{S K}\right)$ vanishes asymptotically; see He et al. (2019a,b). Ignoring $O\left(\frac{1}{S K}\right),(16)$ is a shifting mean which at time $(S k+s) / S K$ can be interpreted as the mean of a stationary AR model whose intercept equals $\delta_{n s}\left(\frac{S k+s}{S K}\right)$ and does not change over time.

\begin{tabular}{rlllll}
\hline & Beijing & Shanghai & St Petersburg & Dublin & Uccle \\
\hline Jan & $* *$ & $*$ & $* * *$ & - & $* *$ \\
Feb & $* * *$ & $* * *$ & - & - & - \\
Mar & $* * *$ & $* * *$ & $* * *$ & $* *$ & $* * *$ \\
Apr & - & $* *$ & $* * *$ & - & $*$ \\
May & $* *$ & $* * *$ & $* * *$ & - & $* *$ \\
Jun & $* *$ & - & - & - & - \\
Jul & - & $* *$ & $*$ & - & - \\
Aug & $* * *$ & $*$ & $*$ & $* *$ & - \\
Sep & $* *$ & $* *$ & - & $* *$ & - \\
Oct & $* *$ & $* * *$ & $* *$ & $* * *$ & $* *$ \\
Nov & $*$ & $* * *$ & $* * *$ & - & $* *$ \\
Dec & $* * *$ & $*$ & $* * *$ & $*$ & $*$
\end{tabular}

Table 2: Results on testing constancy of seasonal means for the five cities. Notation: $p$-value of the test below $0.05\left(^{*}\right)$, below $0.01\left(^{* *}\right)$, and below 0.001 $(* * *)$ 


\subsubsection{Hypothesis testing}

Table 2 contains the results of the constancy tests by city and month. They are summed up by the size of the $p$-value. The values below 0.05 lead to estimating a mean equation in which the corresponding months have a timevarying representation. Beijing has ten $p$-values below 0.05, whereas Dublin has only five.

The estimated seasonal means will be considered next. It should be mentioned that the estimated equations have been tested for error autocorrelation, and the results can be found in Appendix B, Table 8. Where appropriate, the hypothesis of one transition against (at least) two has also been tested using a straightforward extension of the constancy test in Section 4.1. Since there were no $p$-values below 0.05 , the detailed results are not reported here.

\subsubsection{Beijing and Shanghai}

The monthly mean shifts for Beijing can be found in Figure 2 and the ones for Shanghai in Figure 3. The estimated mean equations appear in Tables 3 and 4 . Two technical remarks on the estimated mean equations are in order. For some months, see, for example, October and November in Figure 2 , the estimated seasonal mean is practically a straight line. It is numerically difficult to fit a logistic function to this type of data as there is very little information on where the logistic function is bending, that is, where its second derivative is changing from negative to zero and from zero to positive. As a result, the estimation algorithm may not converge. In these cases, the transition function for equation $n$ and month $j$ is approximated by a straight line:

$$
\sigma_{n j}^{2}\left(\frac{S k+j}{S K}\right)=\delta_{n j 0}+\delta_{n j 1}(S k+j) / S K
$$

In estimating the exponential transition function, uncertainty about the values of $\gamma_{n j 1}, c_{n j 1}$ and $\delta_{n j 1}$ is sometimes also large. In those cases the convergence problem is alleviated by fixing one of the parameters, typically $c_{n j 1}$. Estimates of the other two parameters involved, $\gamma_{n j 1}$ and $\delta_{n j 1}$, are in those cases very strongly negatively correlated, so their standard deviations are remarkably large. See for example estimates for April in Table 3. A similar situation occurs for example in September and December, where the transition function is exponential and the data do not cover the areas where the function is approaching one. Buncic (2019) recently discussed this case which is in fact analogous to the aforementioned situation where the transition function is logistic but no observations at the tails of the function are available. 
It is also seen from Tables 3 and 4 that sometimes the estimate $\widehat{\gamma}_{n j 1}$ is fixed to a high value. When $\gamma_{n j 1}$ is high, its estimate converges slowly to its final value. Since it can be assumed that changes in seasonal means are not completely abrupt, to speed up estimation an upper bound equal to 40 is set for this parameter.

Concerning empirical results, it may be noted that the monthly records begin rather late, in the 1840s. This means that information about effects of the Little Ice Age on monthly temperatures at these two locations is not available. Thus we cannot know whether shifts similar to those reported in European summer temperatures during the 19th century in He et al. (2019a) would have occurred in these two cities. For a discussion of the effects of the Little Ice Age on the climate in China, see Chen, Liu, Zhang, Chen, Huang, Chen, Zhang, Zhou and Chen (2019).

In both series the shifts found occur quite late. Figures 2 and 3 show that they gain strength on the second half of the 20th century. Figure 7 indicates that warming after 1950 has been stronger in Beijing and Shanghai than in the three European locations. The average shifts in the latter series for February, April, October and November are quite substantial, around $3^{\circ} \mathrm{C}$, whereas the size of the corresponding shifts for Beijing remains around half of it. One exception is March, where a record shift of more than $4^{\circ} \mathrm{C}$ is estimated for Beijing. Another one is December. For both locations there is a decrease ending around 1920 or 1930 but, as also seen from Figure 7, the subsequent bounceback is larger for Beijing than for Shanghai.

The increases for both cities are more modest in the summer than in the winter. The timing of the shifts agrees with the increase in Chinese $\mathrm{CO}_{2}$ concentrations. The first notable (positive) change in them occurred about 1970, and the growth accelerated around 2000; see, for example, Zhang and Cheng (2009). Results in Michail, Savva, Koursaros and Zachariadis (2019) suggest that changes in carbon concentrations in the late 1960s or around 1970 have been rather universal.

The only month where the shifts are in the opposite directions is June. However, both are estimated to be small: about $-0.6^{\circ} \mathrm{C}$ for Beijing and $+0.7^{\circ} \mathrm{C}$ for Shanghai. He et al. (2019a) found corresponding negative shifts in June in a number of Northern European series such as Berlin, Stockholm, Uppsala, Vilnius and Warsaw, where their size varied between $1^{\circ} \mathrm{C}$ and $1.5^{\circ} \mathrm{C}$. The typical European shift shape for the summer months, July and August in particular, the shift being first negative and turning positive after 1850, cannot be observed here even if it existed. This is because the Chinese series are too short for that. But then, as already mentioned, both Beijing and Shanghai display a negative December shift that turns positive somewhere between 1920 and 1930 . 
Compared to European series, there is one noteworthy difference. For 17 out of the 20 European series analysed and tested in He et al. (2019a), the null hypothesis of no shift is not rejected for February. On the contrary, a clear shift is found for both Beijing and Shanghai and, as already seen, for the latter it is remarkably large, $3^{\circ} \mathrm{C}$ and for the former close to $2^{\circ} \mathrm{C}$. Whatever the reasons for February being a 'stable' month for European series, these reasons do not seem to be valid either for Beijing or for Shanghai.

Coefficient estimates of the two lags of the temperature variables are low, meaning that the fluctuations in the mean are mainly explained by the time-varying dummy variables. This is also true for equations for the three European locations. Heteroskedasticity-robust tests of no error autocorrelation (not reported) do not reject the null hypothesis for any of the five seasonal mean equations.

\subsubsection{St Petersburg}

The monthly mean shifts for St Petersburg can be found in Figure 4 and the estimated mean equation in Table 5. Figure 7 shows that although warming after 1950 has been generally slower than in either Beijing or Shanghai, it has exceeded that in the other two European locations. The shifts, however, have many similarities to the corresponding shifts in Northern European series in He et al. (2019a). In particular, the rather unusual January shift which begins around 1850 and is over by the end of the century, see Figure 4, can also be found in estimated equations of the VSSMC-AR model for Berlin, Stockholm, Trondheim and Vilnius. Incidentally, it is also found in the well known monthly central England temperature series; see He et al. (2019b). No shift is detected for February, and the same is true for September. Both outcomes may be emphasized because they accord with the results for a majority of the 20 European series in He et al. (2019a). The European summer mean shifts for July and August are clearly visible for St Petersburg. The aforementioned negative June shift for Northern European series is not visible in this series, but for this month no positive shift can be detected either.

There is one similarity between Beijing and St Petersburg. In both locations, the (positive) shift in March is large. As already mentioned, it exceeds $4^{\circ} \mathrm{C}$ for the former station, and the same is true for the latter. This may be a pure coincidence. At any rate, the shift in the St Petersburg series begins earlier than its counterpart for Beijing. It may be noted that the 20 estimated European equations in He et al. (2019a) also display a positive shift for March. Its size, however, is in all cases smaller than that of St Petersburg. Another large positive mean shift, exceeding $4^{\circ} \mathrm{C}$, in the St Petersburg 
series occurs in December, as seen from Figure 4. The estimated European equations have a similar positive shift but, again, it is less strong that the one estimated for St Petersburg.

\subsubsection{Dublin}

For Dublin, the calendar year may be roughly divided into two distinct periods: one extending from January to July and the other from August to December. The estimated mean components can be found in Figure 5 and the corresponding mean equations by month in Table 6 . The shifts considered over three distinct periods are plotted in Figure 7 . In the first period theonly shift appears in March and is less than $1^{\circ} \mathrm{C}$. March is a month in which all estimated European means in He et al. (2019a) contain a positive shift.

The second period contains four small positive shifts (no shift in November). As also seen from Figure 7, none of them begins before 1900. It should be noted that the possible existence of the European summer pattern, a decrease until the second half of the 19th century and a subsequent increase, cannot be sufficiently well observed here even if it existed because the Irish series begins as late as 1831. Of the aforementioned 20 European series, only Paris as the westernmost location in that set shows some similarity to Dublin, although the shifts estimated from the former series are usually larger than they are in the latter. As already noted in Section 5.1.2, Dublin has seven months in which constancy of the mean is not rejected. Paris has six, which is the largest number in the set of 20 stations considered in He et al. (2019a).

It may be concluded that effects of global warming evident in many long European series are less conspicuous in the Dublin one. By comparison it seems that proximity of the Atlantic Ocean has moderated the temperatures and so far precluded big shifts. Obviously, the North Atlantic Oscillation affecting the climate in Europe, see for example Casty, Raible, Stocker, Wanner and Luterbacher (2007), and Asia, Zheng, Li, Li, Zhao and Deng (2016), has had little impact on shifts in monthly average temperatures in Dublin.

\subsubsection{Uccle}

The estimated mean equation for Uccle appears in Table 7. As may be expected, judging from the geographical location, the seasonal patterns for Uccle, depicted in Figure 6, do not deviate very much from those for Dublin. Figure 7 does demonstrate one difference, however. The post-1950 shifts in October-December do not have a counterpart in the estimated Dublin model.

For Uccle, the only shift exceeding $2^{\circ} \mathrm{C}$ occurs in January. The months 
displaying at least a $1^{\circ} \mathrm{C}$ degree shift span a period from October until March, February again being an exception. Thus for the three European locations considered here, no shift is detected for February. For the remaining months Uccle displays either a minor positive shift early in the 20th century (April and May) or no shift. As in the case of Dublin, the measurements begin too late (in 1833) for possible changes in the mean due to the Little Ice Age to be observed and estimated.

\subsection{Shifting seasonal error variances}

\subsubsection{Beijing and Shanghai}

As seen from Figure 8, for the two Asian locations, the seasonal error variances remain low throughout the year and are mostly constant. They are, however, higher in the winter than in the summer. Comparison between the locations shows that there is no systematic difference in the levels between the two cities. For Beijing, the only months for which there is a shift in the variance are July and December, but the timing of this downward shift in them is different. It occurs between 1870 and 1900 for July, whereas the corresponding period extends from about 1960 to 1975 for December. For Shanghai, the only (downward) shift in the error variance can be found in August, and it occurs between 1850 and 1900.

\subsubsection{European locations}

Tests of constancy of error variances (not reported) in the European equations show that the null hypothesis of constancy can be rejected only once at any conventional significance level. There is more variability in the winter than in the summer for each location. St Petersburg differs from the rest in that the variability over the year is much stronger than for the other stations. The errors are not directly comparable with the other stations because the St Petersburg series is much longer than the other four. However, the difference remains even if the first 90 years were excluded from the considerations. A reason for this may be that over the years, St Petersburg has been influenced by different weather conditions: mild winters due to relatively warm air from the Atlantic have been alternating with cold 'Siberian' winters.

Furthermore, stability of the seasonal error variance is not rejected for any month. This variation has not been absorbed by the mean component of the model and consequently shows up in the error variance. It could be argued that winter warming has shifted the St Petersburg distribution, which has increased the dispersion. It is seen from Figure 8, however, that this cannot 
be a major reason because the variation is strongest in February where no mean shift has been detected.

On the contrary, for Dublin, with few exceptions the Atlantic weather patterns have been dominating, which has contributed to a better fit. The difference between winter and summer variances is small. Interestingly, Uccle, located in Western Europe not far from the Atlantic Ocean, lies between these two. The error variances for winter months are higher than in the Dublin equation but are still much lower than the ones for St Petersburg.

The only rejection of constancy occurs in the equation for Uccle, and the month is December. The variance shifts from the relatively high 'January level' closer to the lower 'November level' between about 1925 and 1950 . While there are a few December shifts in the estimated VSSMC-AR model of He et al. (2019a), the timing of the Uccle shift does not match any of them.

\subsection{Shifting seasonal error correlations}

Since the series are not equally long, correlations can only be estimated based on the data from 1847 to 2018 . The model is not re-estimated for this purpose, but its residuals are centred such that they sum up to zero for this particular period. Constancy of correlations (test results not reported) is only rejected for April. For this month, the time-varying correlation matrix is estimated, and likelihood ratio tests are used to sort out the correlations that do not change. Thereafter the correlation matrix with the appropriate restrictions is re-estimated.

The results in Figure 9 show that the European correlations, marked in red, are somewhat higher in the winter months than in the summer. This pattern was also observed in the dataset analysed by He et al. (2019a). The only exception to this rule is the Dublin-Uccle correlation which is rather steady and only fluctuates between 0.6 and 0.7 throughout the year. The only Asian, that is, the Beijing-Shanghai correlation also fits this pattern. For these two pairs it seems that the variation not captured by the seasonal means contains factors that are common for them. In April, however, both correlations surge towards unity at the end of the period. The correlation between St Petersburg and Uccle is generally lowest in this group but remains slightly positive for all 12 months.

The monthly 'intercontinental' correlations involving one Asian and one European location, marked in blue in Figure 9, are mostly positive and close to zero or slightly negative throughout the year. In April, the correlations between Shanghai on the one hand and both Dublin and Uccle on the other become strongly negative towards the end of the period. Overall, the Shanghai- 
St Petersburg correlation, which is also the one between the northernmost and the southernmost city in this dataset, is generally the lowest one, always constant and slightly negative. It appears that the temperature movements not accommodated in the seasonal means and variances are rather unrelated across continents.

\section{Final remarks}

In this paper the VSSMC-AR model is applied to long monthly temperature series from five cities, two from Asia (China) and three from Europe. The interest centres on two issues: differences between shifts in the Asian and European series and those between 'Atlantic' and continental European ones. The estimation results for seasonal means show that warming in Beijing and Shanghai on the average has begun later than in Europe, and it has been stronger in the winter than in the summer. As to seasonal error variances from the model, they are smaller than for Uccle or St Petersburg but in line with those from the Dublin equation.

Among the three European series, there are clear differences in warming. The estimated equation for Dublin shows that warming has so far been rather modest throughout the year. For seven months, no warming trend has been detected. Compared to St Petersburg, warming in Uccle has also been rather minor, and for five months, constancy of the seasonal mean has not been rejected.

Estimated error correlations suggest that factors not explained by the seasonal means and variances are different in Asia (Beijing and Shanghai) from Europe. The intercontinental correlations are either positive and low or slightly negative. The strongest correlation is that between the 'neighbouring' cities Dublin and Uccle, and the other correlation separate from the rest is the one between Beijing and Shanghai. It may be concluded, not unexpectedly, that the distance between the locations affects the correlations. He et al. (2019a) arrived at a similar conclusion, but they also did notice that geography plays a role.

It would be interesting to add more Asian cities to this comparison. A drawback would be that to the best of our knowledge the other monthly Asian temperature series would be shorter than the two series analysed here and/or would contain a rather large number of missing values. This could diminish strength of the conclusions, although such an exercise would certainly not be worthless. It is nevertheless left for further research. 


\section{References}

Berben, R.-P. and Jansen, W. J.: 2005, Comovement in international equity markets: A sectoral view, Journal of International Money and Finance 24, 832-857.

Buncic, D.: 2019, Identification and estimation issues in exponential smooth transition autoregressive models, Oxford Bulletin of Economics and Statistics 81, 667-685.

Casty, C., Raible, C. C., Stocker, T. F., Wanner, H. and Luterbacher, J.: 2007, A European pattern climatology 1766-2000, Climate Dynamics 29, 791-805.

Chen, J., Liu, J., Zhang, X., Chen, S., Huang, W., Chen, J., Zhang, S., Zhou, A. and Chen, F.: 2019, Unstable Little Ice Age climate revealed by highresolution proxy records from northwestern China, Climate Dynamics 53, 1517-1546.

González, A. and Teräsvirta, T.: 2008, Modelling autoregressive processes with a shifting mean, Studies in Nonlinear Dynamics and Econometrics 12, Iss. 1, Article 1.

He, C., Kang, J., Teräsvirta, T. and Zhang, S.: 2019a, Long monthly temperature series and the vector seasonal shifting mean and covariance autoregressive model, CREATES Research Paper 2019-18, Aarhus University.

He, C., Kang, J., Teräsvirta, T. and Zhang, S.: 2019b, The shifting seasonal mean autoregressive model and seasonality in the central England monthly temperature series, 1772-2016, Econometrics and Statistics 12, 1-24.

Hillebrand, E. and Proietti, T.: 2017, Phase changes and seasonal warming in early instrumental temperature records, Journal of Climate 30, 67956821.

Lin, C.-F. J. and Teräsvirta, T.: 1994, Testing the constancy of regression parameters against continuous structural change, Journal of Econometrics 62, 211-228.

Luukkonen, R., Saikkonen, P. and Teräsvirta, T.: 1988, Testing linearity against smooth transition autoregressive models, Biometrika 75, 491499. 
Michail, N. A., Savva, C. S., Koursaros, D. and Zachariadis, T.: 2019, Anthropogenic greenhouse gas concentrations and global temperature: a smooth transition analysis, $S N$ Applied Sciences 1, https://doi.org/10.1007/s42452-019-0670-6.

Saikkonen, P. and Luukkonen, R.: 1988, Lagrange multiplier tests for testing nonlinearities in time series models, Scandinavian Journal of Statistics 15, 55-68.

Silvennoinen, A. and Teräsvirta, T.: 2005, Multivariate autoregressive conditional heteroskedasticity with smooth transitions in conditional correlations, SSE/EFI Working Paper Series in Economics and Finance No. 577.

Silvennoinen, A. and Teräsvirta, T.: 2015, Modeling conditional correlations of asset returns: A smooth transition approach, Econometric Reviews 34, 174-197.

Silvennoinen, A. and Teräsvirta, T.: 2016, Testing constancy of unconditional variance in volatility models by misspecification and specification tests, Studies in Nonlinear Dynamics and Econometrics 20, 347-364.

Teräsvirta, T.: 1994, Specification, estimation, and evaluation of smooth transition autoregressive models, Journal of the American Statistical Association 89, 208-218.

Wooldridge, J. M.: 1990, A unified approach to robust, regression-based specification tests, Econometric Theory 6, 17-43.

Zhang, X.-P. and Cheng, X.-M.: 2009, Energy consumption, carbon emissions, and economic growth in China, Ecological Economics 68, 27062712 .

Zheng, F., Li, J., Li, Y., Zhao, S. and Deng, D.: 2016, Influence of the summer $\mathrm{NAO}$ on the spring-NAO-based predictability of the East Asian summer monsoon, Journal of Applied Meteorology and Climatology 55, 14591476. 


\section{A Figures}

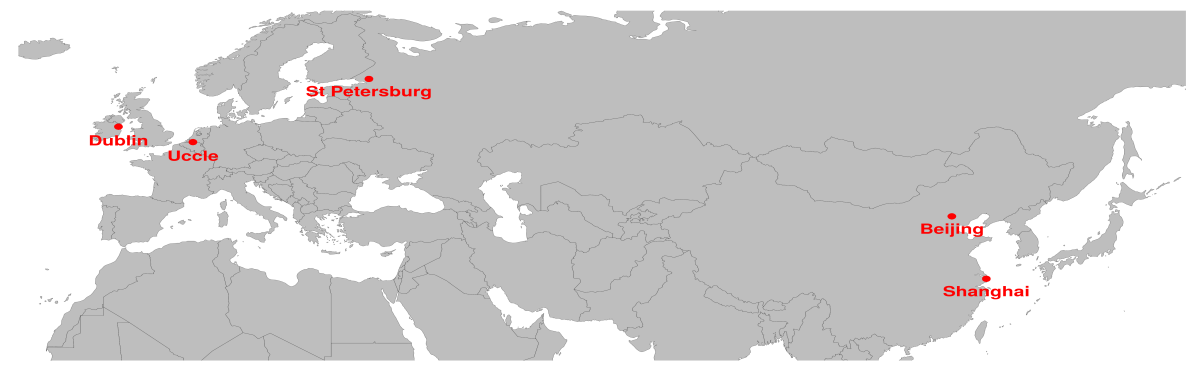

Figure 1. Locations of the five cities, Beijing, Shanghai, St Petersburg, Dublin and Uccle 

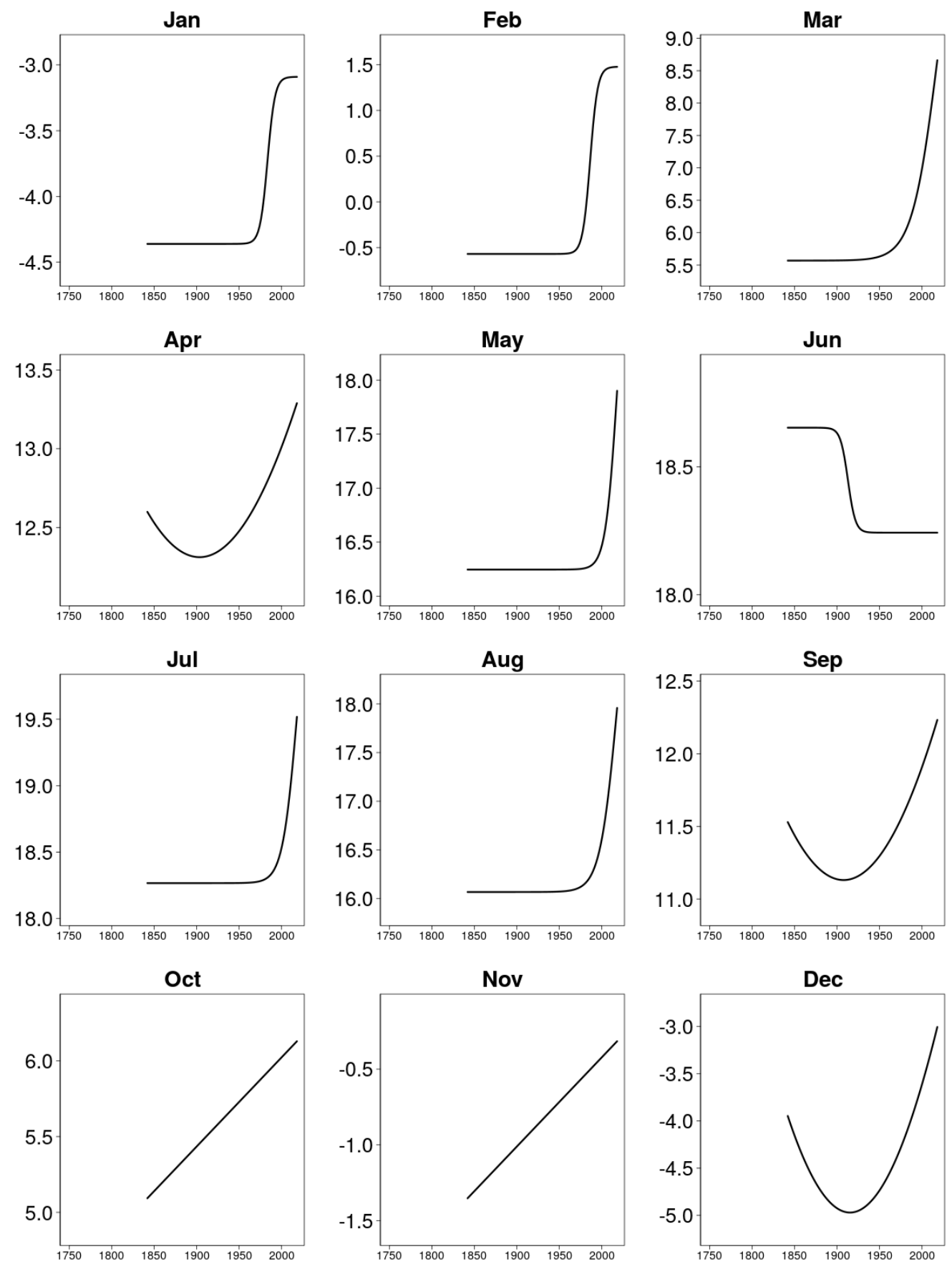

Figure 2. Shifting average monthly temperatures in Beijing, 1841-2018, estimated from the model (1) 

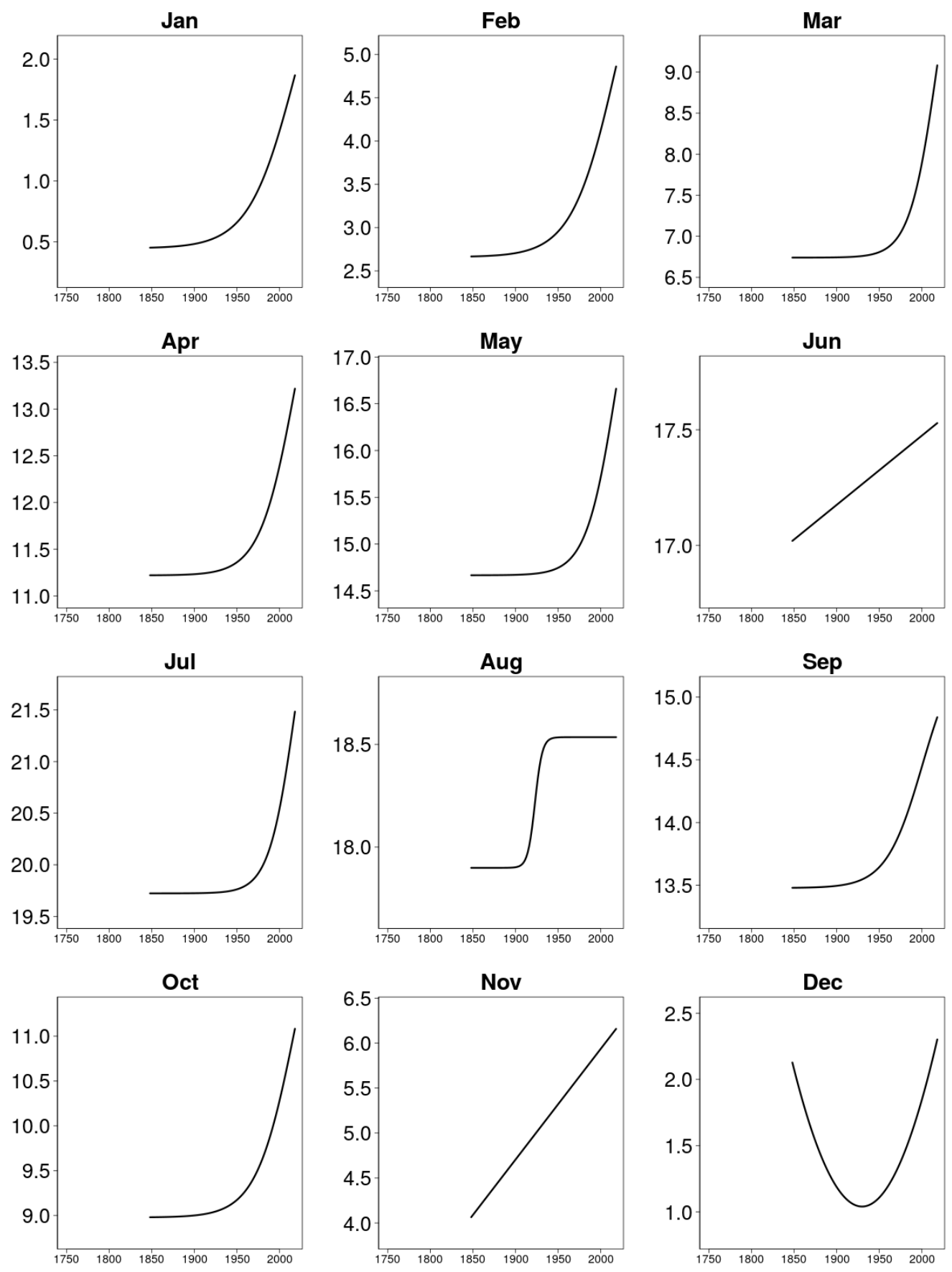

Figure 3. Shifting average monthly temperatures in Shanghai, 1847-2018, estimated from the VSSMC-AR model (1) 

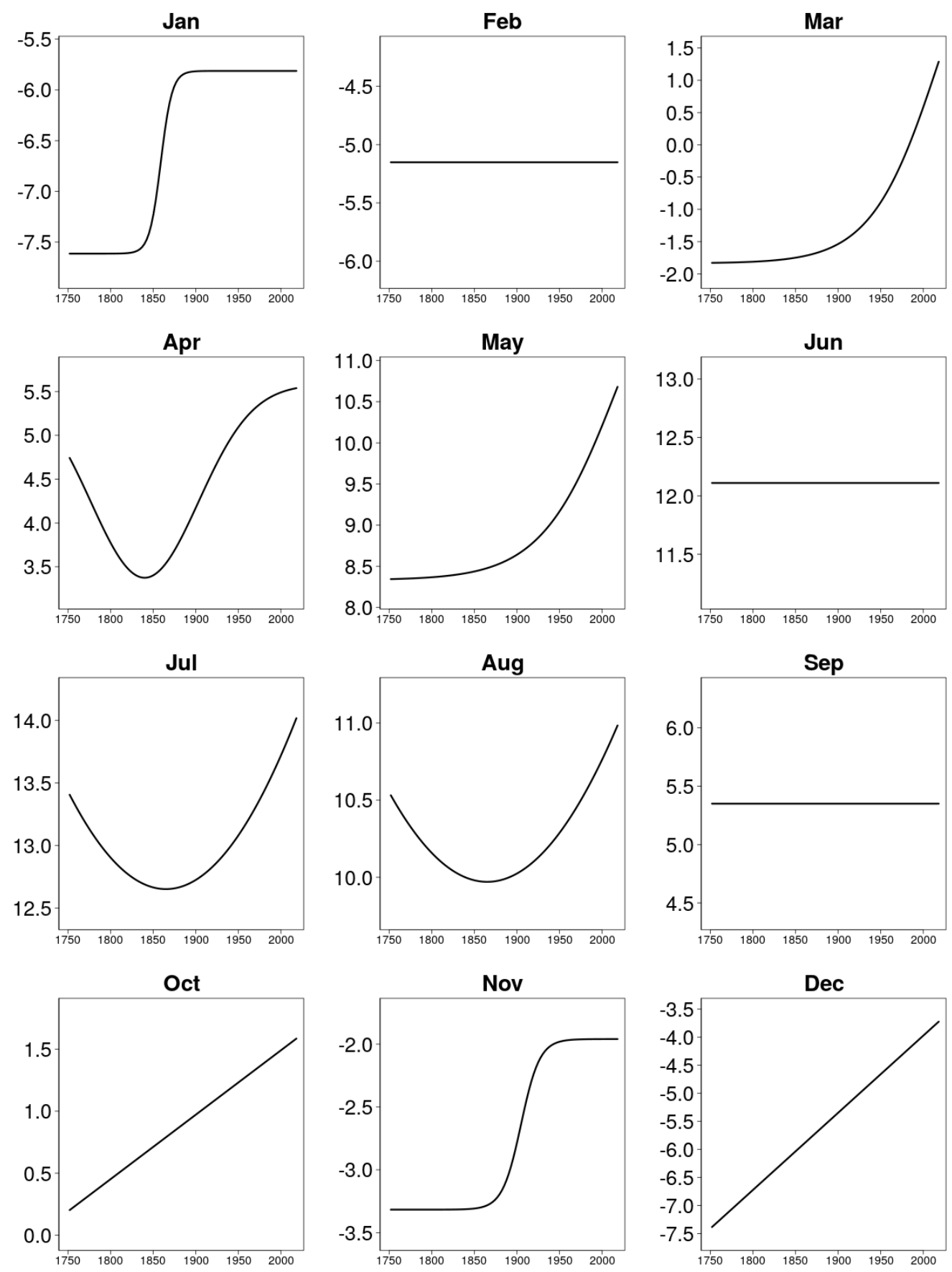

Figure 4. Shifting average monthly temperatures in St Petersburg, 1752-2018, estimated from the VSSMC-AR model (1) 

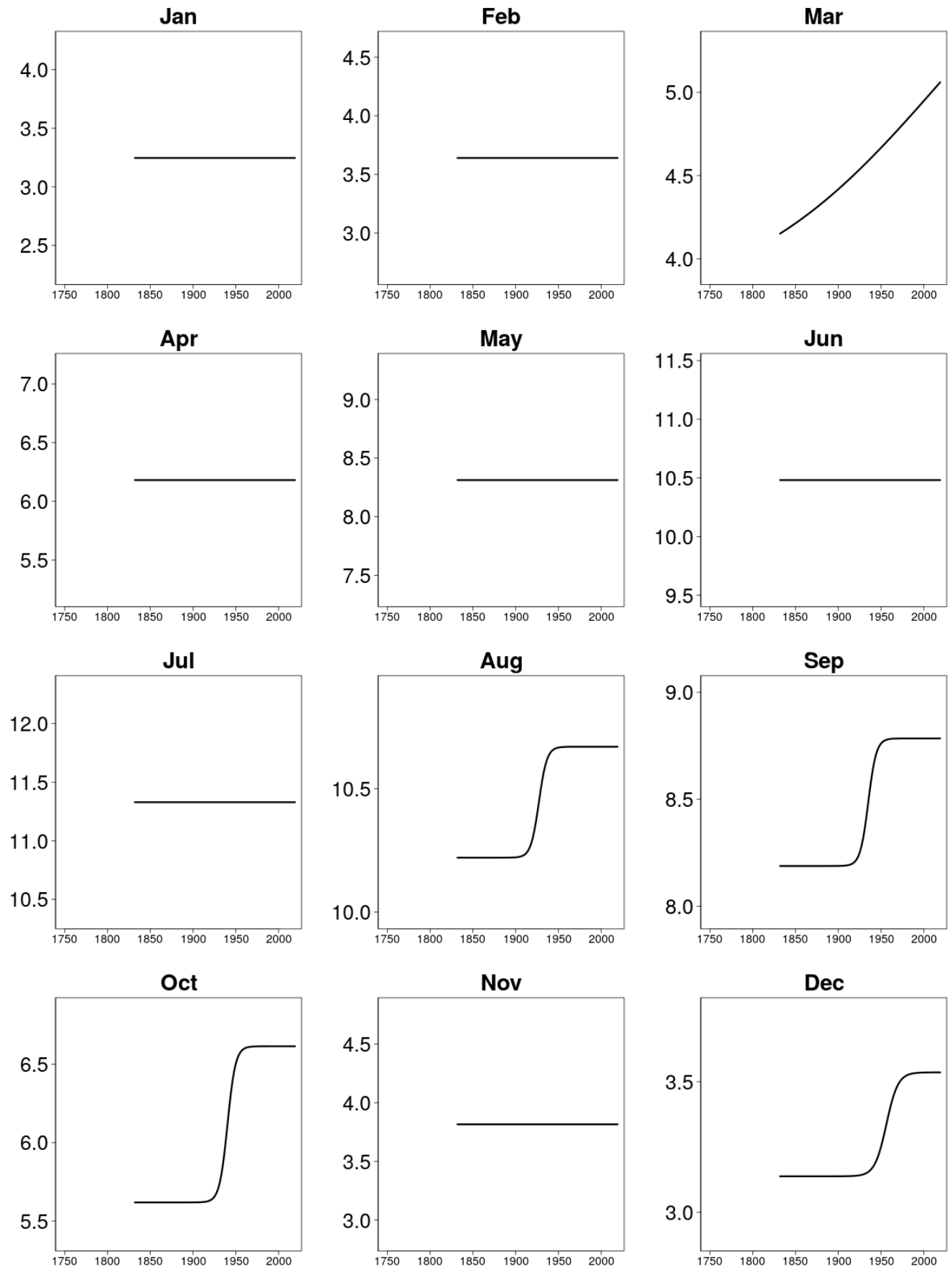

Figure 5. Shifting average monthly temperatures in Dublin, 1831-2018, estimated from the VSSMC-AR model (1) 

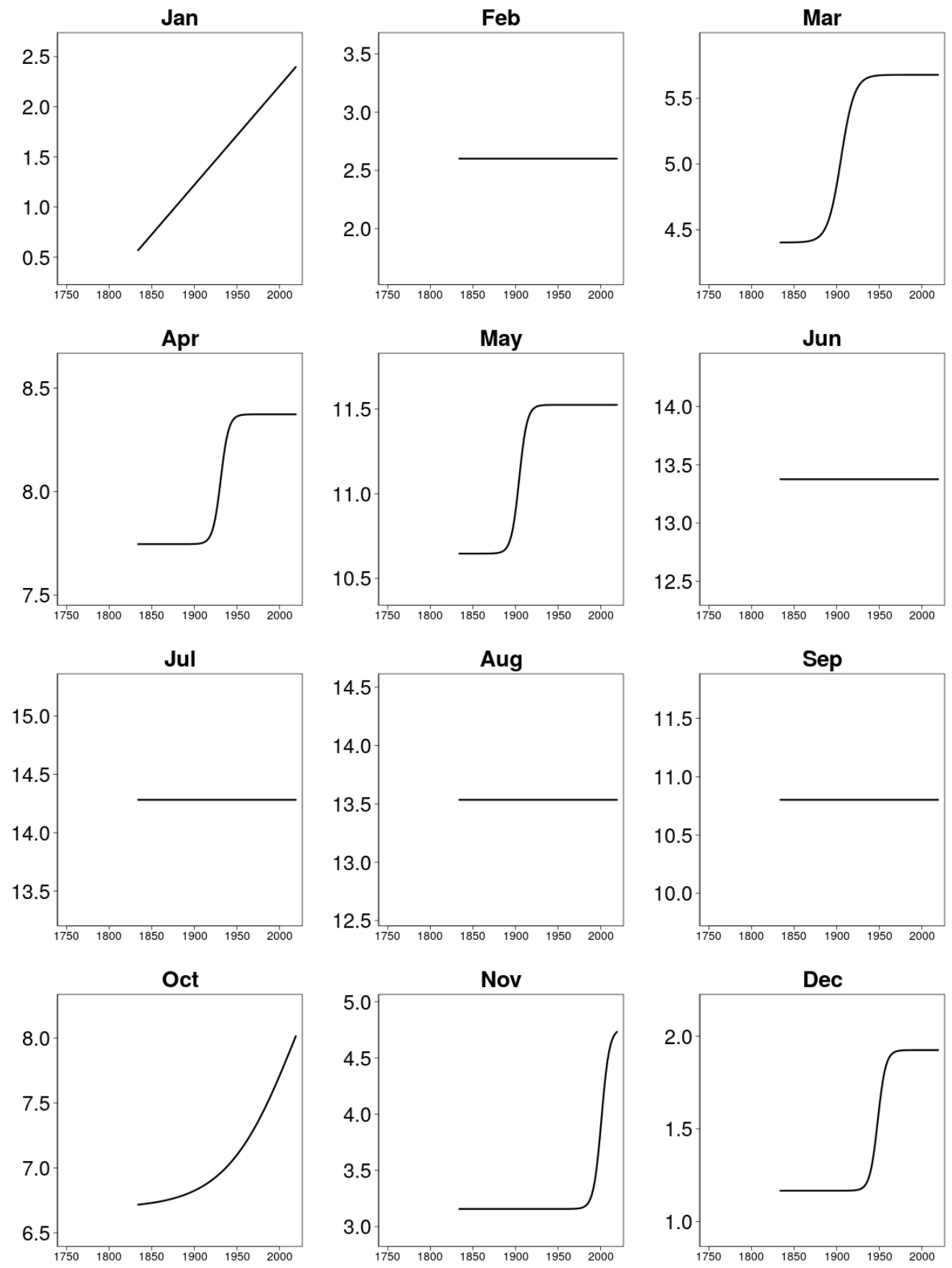

Figure 6. Shifting average monthly temperatures in Uccle, 1833-2018, estimated from the VSSMC-AR model (1) 

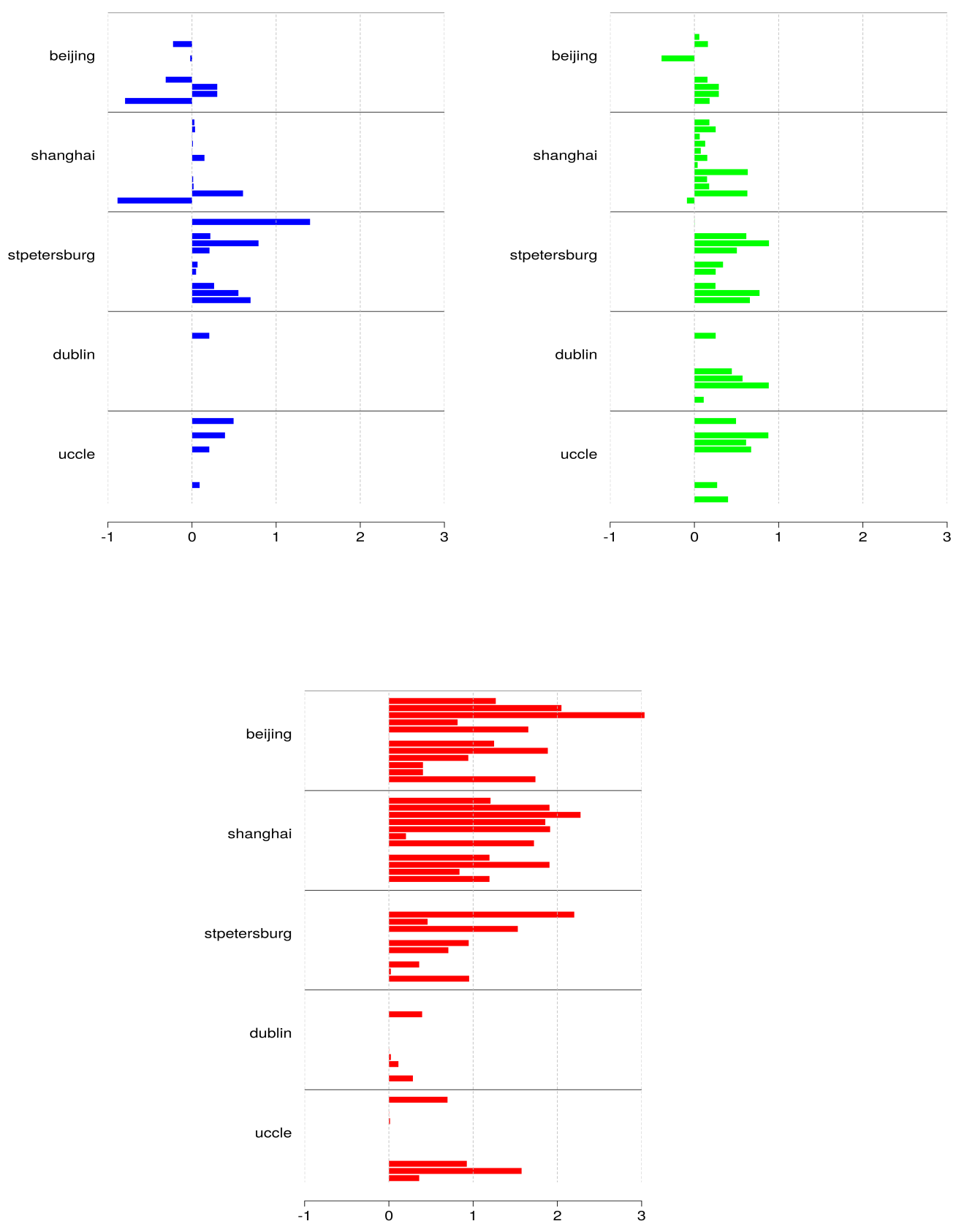

Figure 7. Plots of estimated shift sizes allocated on periods 1850-1900, 1900-1950 and 1950-2018 for the five locations ( $x$-axis measures the shift in centigrade). 

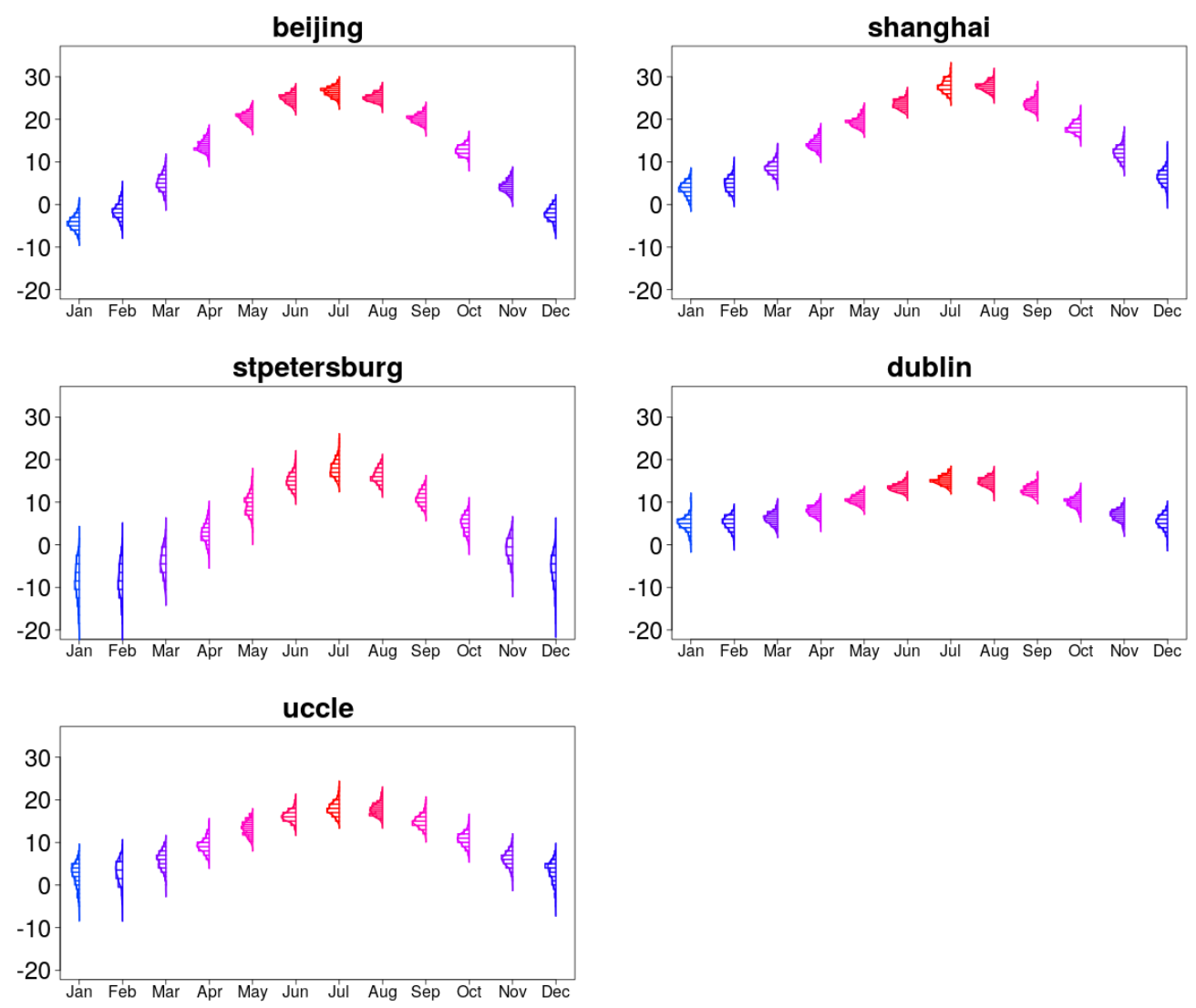

Figure 8. Histograms of the estimated error variances from the VSSMC-AR model for the five cities 

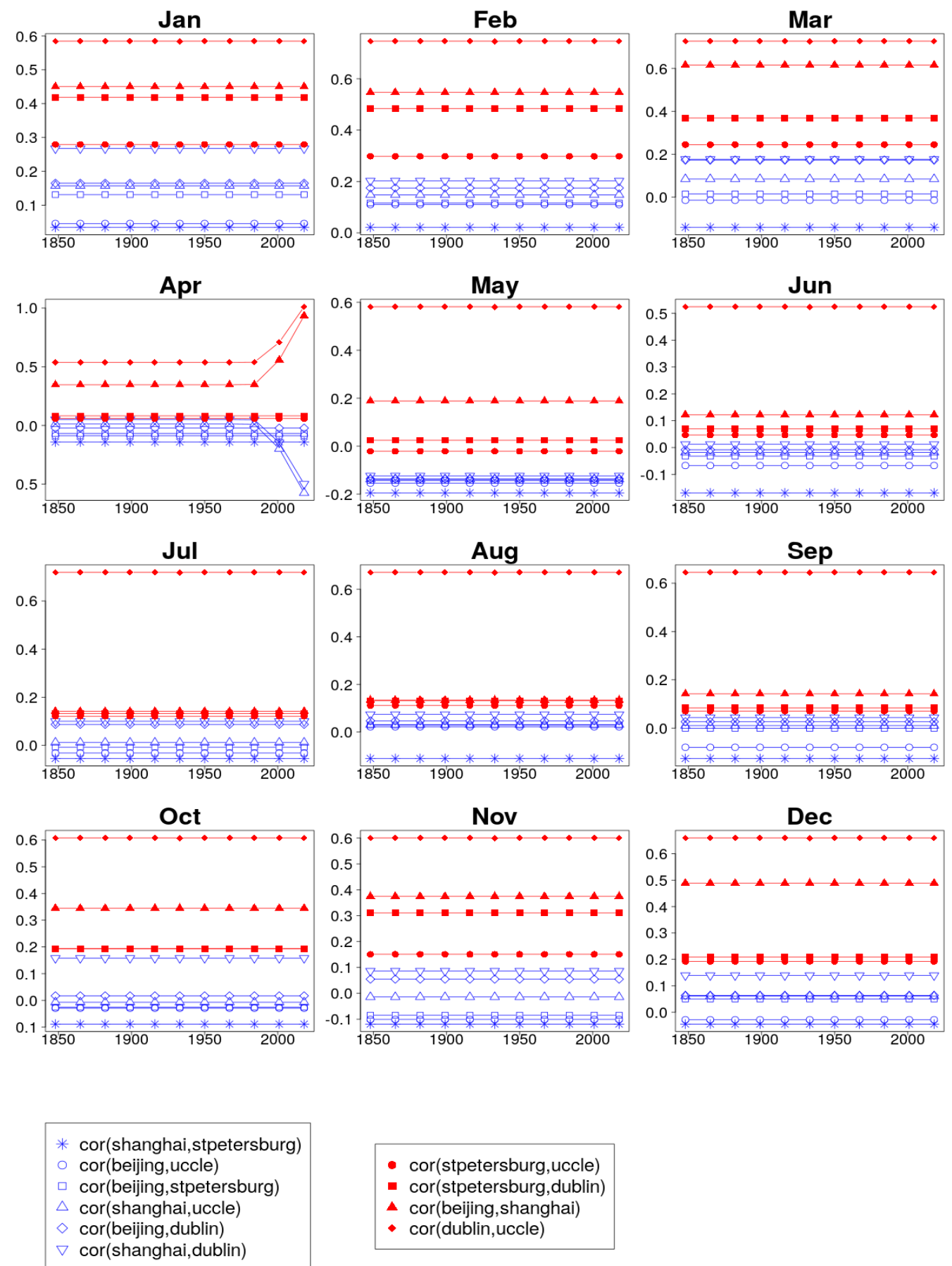

- cor(stpetersburg,uccle)

- cor(stpetersburg,dublin)

- cor(beijing,shanghai)

- cor(dublin,uccle)

Figure 9. Estimated error correlations from the VSSMC-AR model for temperatures of the five cities, 1847-2018 


\section{B Tables}

This appendix contains the tables for the five estimated mean equations. Three remarks are in order. First, as has been mentioned in Section 5.1.3, sometimes it has not been numerically possible to estimate the logistic transition function because there has not been enough information in the data about where the function bends. It has then been replaced by a linear trend. These cases are distinguished in the table by the fact that there are no estimates for either the slope parameter $\gamma_{j}$ or location parameter $c_{j}$. The two parameter estimates, $\widehat{\delta}_{j 0}$ and $\widehat{\delta}_{j 1}$, are the intercept and the coefficient of the linear trend $t / T$. Furthermore, in the absence of a shift, only $\widehat{\delta}_{j 0}$ has an estimate. Second, in some cases the transition is rapid, and the slope parameter $\gamma_{j}$ has been fixed to the value 40 that has functioned as the upper bound. This is why the 'estimate' lacks a standard deviation estimate. Third, in the estimation of an exponential transition function, $c_{j}$ has sometimes been fixed, typically to unity. At the same time, the standard deviations of $\widehat{\gamma}_{j}$ and $\widehat{\delta}_{j 1}$ remain very large because these estimates are strongly negative correlated and their joint uncertainty is thus large. As discussed in Section 5.1.3, the reason for this is that there has not been enough information in the data to cover the part of the exponential transition function where it begins to approach its limiting value unity. As a result, it has not been numerically possible to obtain estimates for these three parameters simultaneously. 


\begin{tabular}{lrrlrr}
\hline Parameter & Estimate & Std. Error & Parameter & Estimate & Std. Error \\
\hline$\phi_{1}$ & 0.256 & 0.022 & $c_{6}$ & 0.402 & 0.092 \\
$\phi_{2}$ & 0.084 & 0.015 & $\delta_{7,0}$ & 18.267 & 0.592 \\
$\delta_{1,0}$ & -4.361 & 0.156 & $\delta_{7,1}$ & 2.501 & 1.223 \\
$\delta_{1,1}$ & 1.271 & 0.268 & $\gamma_{7}$ & 20.786 & 14.638 \\
$\gamma_{1}$ & 40.000 & - & $c_{7}$ & 1.000 & - \\
$c_{1}$ & 0.804 & 0.032 & $\delta_{8,0}$ & 16.068 & 0.667 \\
$\delta_{2,0}$ & -0.570 & 0.145 & $\delta_{8,1}$ & 3.780 & 1.115 \\
$\delta_{2,1}$ & 2.050 & 0.283 & $\gamma_{8}$ & 17.442 & 7.535 \\
$\gamma_{2}$ & 40.000 & - & $c_{8}$ & 1.000 & - \\
$c_{2}$ & 0.820 & 0.020 & $\delta_{9,0}$ & 11.131 & 0.683 \\
$\delta_{3,0}$ & 5.568 & 0.152 & $\delta_{9,1}$ & 21.427 & 921.697 \\
$\delta_{3,1}$ & 6.189 & 0.967 & $\gamma_{9}$ & 0.135 & 5.914 \\
$\gamma_{3}$ & 11.857 & 2.769 & $c_{9}$ & 0.374 & 0.077 \\
$c_{3}$ & 1.000 & - & $\delta_{10,0}$ & 5.093 & 0.603 \\
$\delta_{4,0}$ & 12.312 & 0.197 & $\delta_{10,1}$ & 1.035 & 0.311 \\
$\delta_{4,1}$ & 15.874 & 648.736 & $\gamma_{10}$ & & - \\
$\gamma_{4}$ & 0.150 & 6.293 & $c_{10}$ & - & - \\
$c_{4}$ & 0.349 & 0.101 & $\delta_{11,0}$ & -1.352 & 0.464 \\
$\delta_{5,0}$ & 16.246 & 0.298 & $\delta_{11,1}$ & 1.037 & 0.315 \\
$\delta_{5,1}$ & 3.310 & 1.390 & $\gamma_{11}$ & - & - \\
$\gamma_{5}$ & 25.786 & 15.300 & $c_{11}$ & - & - \\
$c_{5}$ & 1.000 & - & $\delta_{12,0}$ & -4.970 & 0.310 \\
$\delta_{6,0}$ & 18.653 & 0.476 & $\delta_{12,1}$ & 20.861 & 275.498 \\
$\delta_{6,1}$ & -0.411 & 0.195 & $\gamma_{12}$ & 0.290 & 3.978 \\
$\gamma_{6}$ & 40.000 & - & $c_{12}$ & 0.416 & 0.033 \\
\hline
\end{tabular}

Table 3: Estimated monthly seasonal mean equation for Beijing. Note: See explanations in the beginning of the appendix for notation 


\begin{tabular}{lrrlrr}
\hline Parameter & Estimate & Std. Error & Parameter & Estimate & Std. Error \\
\hline$\phi_{1}$ & 0.237 & 0.022 & $c_{6}$ & - & - \\
$\phi_{2}$ & 0.115 & 0.017 & $\delta_{7,0}$ & 19.723 & 0.587 \\
$\delta_{1,0}$ & 0.447 & 0.300 & $\delta_{7,1}$ & 3.521 & 0.903 \\
$\delta_{1,1}$ & 2.841 & 0.709 & $\gamma_{7}$ & 11.223 & 4.385 \\
$\gamma_{1}$ & 6.281 & 2.852 & $c_{7}$ & 1.000 & - \\
$c_{1}$ & 1.000 & - & $\delta_{8,0}$ & 17.899 & 0.706 \\
$\delta_{2,0}$ & 2.661 & 0.206 & $\delta_{8,1}$ & 0.637 & 0.189 \\
$\delta_{2,1}$ & 4.402 & 0.717 & $\gamma_{8}$ & 40.000 & - \\
$\gamma_{2}$ & 6.582 & 1.917 & $c_{8}$ & 0.440 & 0.058 \\
$c_{2}$ & 1.000 & - & $\delta_{9,0}$ & 13.478 & 0.757 \\
$\delta_{3,0}$ & 6.741 & 0.155 & $\delta_{9,1}$ & 1.961 & 3.224 \\
$\delta_{3,1}$ & 4.684 & 0.897 & $\gamma_{9}$ & 7.993 & 9.968 \\
$\gamma_{3}$ & 10.566 & 3.085 & $c_{9}$ & 0.897 & 0.422 \\
$c_{3}$ & 1.000 & - & $\delta_{10,0}$ & 8.979 & 0.702 \\
$\delta_{4,0}$ & 11.219 & 0.222 & $\delta_{10,1}$ & 4.207 & 0.739 \\
$\delta_{4,1}$ & 3.997 & 0.800 & $\gamma_{10}$ & 7.549 & 2.266 \\
$\gamma_{4}$ & 8.270 & 2.667 & $c_{10}$ & 1.000 & - \\
$c_{4}$ & 1.000 & - & $\delta_{11,0}$ & 4.065 & 0.579 \\
$\delta_{5,0}$ & 14.666 & 0.333 & $\delta_{11,1}$ & 2.094 & 0.311 \\
$\delta_{5,1}$ & 3.989 & 0.860 & $\gamma_{11}$ & - & - \\
$\gamma_{5}$ & 9.715 & 3.235 & $c_{11}$ & - & - \\
$c_{5}$ & 1.000 & - & $\delta_{12,0}$ & 1.040 & 0.446 \\
$\delta_{6,0}$ & 17.019 & 0.469 & $\delta_{12,1}$ & 22.510 & 791.349 \\
$\delta_{6,1}$ & 0.510 & 0.311 & $\gamma_{12}$ & 0.214 & 7.702 \\
$\gamma_{6}$ & - & - & $c_{12}$ & 0.481 & 0.034 \\
\hline
\end{tabular}

Table 4: Estimated monthly seasonal mean equation for Shanghai. Note: See explanations in the beginning of the appendix for notation 


\begin{tabular}{lrrlrr}
\hline Parameter & Estimate & Std. Error & Parameter & Estimate & Std. Error \\
\hline$\phi_{1}$ & 0.291 & 0.018 & $c_{6}$ & - & - \\
$\phi_{2}$ & 0.051 & 0.014 & $\delta_{7,0}$ & 12.651 & 0.365 \\
$\delta_{1,0}$ & -7.613 & 0.280 & $\delta_{7,1}$ & 24.757 & 1364.012 \\
$\delta_{1,1}$ & 1.800 & 0.324 & $\gamma_{7}$ & 0.171 & 9.650 \\
$\gamma_{1}$ & 40.000 & - & $c_{7}$ & 0.425 & 0.076 \\
$c_{1}$ & 0.402 & 0.035 & $\delta_{8,0}$ & 9.970 & 0.423 \\
$\delta_{2,0}$ & -5.152 & 0.208 & $\delta_{8,1}$ & 20.494 & 1699.677 \\
$\delta_{2,1}$ & - & - & $\gamma_{8}$ & 0.154 & 12.989 \\
$\gamma_{2}$ & - & - & $c_{8}$ & 0.425 & 0.102 \\
$c_{2}$ & - & - & $\delta_{9,0}$ & 5.350 & 0.381 \\
$\delta_{3,0}$ & -1.837 & 0.314 & $\delta_{9,1}$ & - & - \\
$\delta_{3,1}$ & 6.250 & 1.216 & $\gamma_{9}$ & - & - \\
$\gamma_{3}$ & 6.738 & 2.323 & $c_{9}$ & - & - \\
$c_{3}$ & 1.000 & - & $\delta_{10,0}$ & 0.202 & 0.409 \\
$\delta_{4,0}$ & 3.375 & 0.336 & $\delta_{10,1}$ & 1.384 & 0.513 \\
$\delta_{4,1}$ & 2.205 & 0.522 & $\gamma_{10}$ & - & - \\
$\gamma_{4}$ & 8.846 & 5.675 & $c_{10}$ & - & - \\
$c_{4}$ & 0.331 & 0.039 & $\delta_{11,0}$ & -3.317 & 0.292 \\
$\delta_{5,0}$ & 8.332 & 0.299 & $\delta_{11,1}$ & 1.356 & 0.379 \\
$\delta_{5,1}$ & 4.698 & 1.151 & $\gamma_{11}$ & 24.403 & 32.623 \\
$\gamma_{5}$ & 5.987 & 2.772 & $c_{11}$ & 0.574 & 0.063 \\
$c_{5}$ & 1.000 & - & $\delta_{12,0}$ & -7.385 & 0.310 \\
$\delta_{6,0}$ & 12.110 & 0.218 & $\delta_{12,1}$ & 3.661 & 0.518 \\
$\delta_{6,1}$ & - & - & $\gamma_{12}$ & - & - \\
$\gamma_{6}$ & - & - & $c_{12}$ & - & - \\
\hline & & & & & \\
& & - & & & - \\
\hline
\end{tabular}

Table 5: Estimated monthly seasonal mean equation for St Petersburg. Note: See explanations in the beginning of the appendix for notation. 


\begin{tabular}{lrrlrr}
\hline Parameter & Estimate & Std. Error & Parameter & Estimate & Std. Error \\
\hline$\phi_{1}$ & 0.227 & 0.021 & $c_{6}$ & - & - \\
$\phi_{2}$ & 0.063 & 0.023 & $\delta_{7,0}$ & 11.329 & 0.323 \\
$\delta_{1,0}$ & 3.246 & 0.182 & $\delta_{7,1}$ & - & - \\
$\delta_{1,1}$ & - & - & $\gamma_{7}$ & - & - \\
$\gamma_{1}$ & - & - & $c_{7}$ & - & - \\
$c_{1}$ & - & - & $\delta_{8,0}$ & 10.220 & 0.386 \\
$\delta_{2,0}$ & 3.640 & 0.159 & $\delta_{8,1}$ & 0.449 & 0.181 \\
$\delta_{2,1}$ & - & - & $\gamma_{8}$ & 40.000 & - \\
$\gamma_{2}$ & - & - & $c_{8}$ & 0.508 & 0.080 \\
$c_{2}$ & - & - & $\delta_{9,0}$ & 8.188 & 0.397 \\
$\delta_{3,0}$ & 3.691 & 2.431 & $\delta_{9,1}$ & 0.597 & 0.182 \\
$\delta_{3,1}$ & 2.740 & 4.580 & $\gamma_{9}$ & 40.000 & - \\
$\gamma_{3}$ & 1.601 & 3.792 & $c_{9}$ & 0.551 & 0.060 \\
$c_{3}$ & 1.000 & - & $\delta_{10,0}$ & 5.618 & 0.368 \\
$\delta_{4,0}$ & 6.181 & 0.168 & $\delta_{10,1}$ & 0.997 & 0.184 \\
$\delta_{4,1}$ & - & - & $\gamma_{10}$ & 40.000 & - \\
$\gamma_{4}$ & - & - & $c_{10}$ & 0.579 & 0.036 \\
$c_{4}$ & - & - & $\delta_{11,0}$ & 3.816 & 0.308 \\
$\delta_{5,0}$ & 8.312 & 0.201 & $\delta_{11,1}$ & & - \\
$\delta_{5,1}$ & - & - & $\gamma_{11}$ & - & - \\
$\gamma_{5}$ & - & - & $c_{11}$ & - & - \\
$c_{5}$ & 10.481 & 0.255 & $\delta_{12,1}$ & 0.398 & 0.232 \\
$\delta_{6,0}$ & - & - & $\gamma_{12}$ & 27.639 & 77.039 \\
$\delta_{6,1}$ & - & - & $c_{12}$ & 0.664 & 0.116 \\
$\gamma_{6}$ & & & & & - \\
\hline
\end{tabular}

Table 6: Estimated monthly seasonal mean equation for Dublin. Note: See explanations in the beginning of the appendix for notation 


\begin{tabular}{lrrlrr}
\hline Parameter & Estimate & Std. Error & Parameter & Estimate & Std. Error \\
\hline$\phi_{1}$ & 0.173 & 0.021 & $c_{6}$ & - & - \\
$\phi_{2}$ & 0.062 & 0.013 & $\delta_{7,0}$ & 14.282 & 0.418 \\
$\delta_{1,0}$ & 0.568 & 0.282 & $\delta_{7,1}$ & - & - \\
$\delta_{1,1}$ & 1.829 & 0.447 & $\gamma_{7}$ & - & - \\
$\gamma_{1}$ & - & - & $c_{7}$ & - & - \\
$c_{1}$ & - & - & $\delta_{8,0}$ & 13.535 & 0.477 \\
$\delta_{2,0}$ & 2.601 & 0.149 & $\delta_{8,1}$ & - & - \\
$\delta_{2,1}$ & - & - & $\gamma_{8}$ & - & - \\
$\gamma_{2}$ & - & - & $c_{8}$ & - & - \\
$c_{2}$ & - & - & $\delta_{9,0}$ & 10.802 & 0.494 \\
$\delta_{3,0}$ & 4.403 & 0.276 & $\delta_{9,1}$ & - & - \\
$\delta_{3,1}$ & 1.275 & 0.342 & $\gamma_{9}$ & - & - \\
$\gamma_{3}$ & 23.740 & 29.155 & $c_{9}$ & - & - \\
$c_{3}$ & 0.384 & 0.059 & $\delta_{10,0}$ & 6.690 & 0.554 \\
$\delta_{4,0}$ & 7.747 & 0.223 & $\delta_{10,1}$ & 2.650 & 0.908 \\
$\delta_{4,1}$ & 0.627 & 0.272 & $\gamma_{10}$ & 4.543 & 3.554 \\
$\gamma_{4}$ & 40.000 & - & $c_{10}$ & 1.000 & - \\
$c_{4}$ & 0.524 & 0.086 & $\delta_{11,0}$ & 3.157 & 0.378 \\
$\delta_{5,0}$ & 10.646 & 0.301 & $\delta_{11,1}$ & 1.605 & 0.646 \\
$\delta_{5,1}$ & 0.879 & 0.283 & $\gamma_{11}$ & 40.000 & - \\
$\gamma_{5}$ & 40.000 & - & $c_{11}$ & 0.902 & 0.043 \\
$c_{5}$ & 0.380 & 0.062 & $\delta_{12,0}$ & 1.167 & 0.289 \\
$\delta_{6,0}$ & 13.376 & 0.334 & $\delta_{12,1}$ & 0.758 & 0.281 \\
$\delta_{6,1}$ & - & - & $\gamma_{12}$ & 40.000 & - \\
$\gamma_{6}$ & - & - & $c_{12}$ & 0.617 & 0.072 \\
\hline
\end{tabular}

Table 7: Estimated monthly seasonal mean equation for Uccle. Note: See explanations in the beginning of the appendix for notation 


\begin{tabular}{rrrrrr}
\hline & $p \mathrm{AR}(1)$ & $p \mathrm{AR}(2)$ & $p \mathrm{AR}(3)$ & $p \mathrm{AR}(6)$ & $p \mathrm{AR}(12)$ \\
\hline Beijing & 0.710 & 0.719 & 0.488 & 0.688 & 0.345 \\
Shanghai & 0.915 & 0.855 & 0.610 & 0.547 & 0.231 \\
St Petersburg & 0.659 & 0.544 & 0.505 & 0.683 & 0.460 \\
Dublin & 0.621 & 0.652 & 0.362 & 0.314 & 0.064 \\
Uccle & 0.940 & 0.890 & 0.942 & 0.384 & 0.222 \\
\hline
\end{tabular}

Table 8: $p$-values of the test of no error autocorrelation for the five seasonal mean equations in the estimated VSSMC-AR model against error autocorrelations of various length 
2019-03: Martin M. Andreasen and Mads Dang: Estimating the Price Markup in the New Keynesian Model

2019-04: Daniel Borup, Bent Jesper Christensen and Yunus Emre Ergemen: Assessing predictive accuracy in panel data models with long-range dependence

2019-05: $\quad$ Antoine A. Djogbenou, James G. MacKinnon and Morten Ørregaard Nielsen: Asymptotic Theory and Wild Bootstrap Inference with Clustered Errors

2019-06: Vanessa Berenguer-Rico, Søren Johansen and Bent Nielsen: The analysis of marked and weighted empirical processes of estimated residuals

2019-07: $\quad$ Søren Kjærgaard, Yunus Emre Ergemen, Kallestrup-Lamb, Jim Oeppen and Rune Lindahl-Jacobsen: Forecasting Causes of Death using Compositional Data Analysis: the Case of Cancer Deaths

2019-08: $\quad$ Søren Kjærgaard, Yunus Emre Ergemen, Marie-Pier Bergeron Boucher, Jim Oeppen and Malene Kallestrup-Lamb: Longevity forecasting by socio-economic groups using compositional data analysis

2019-09: Debopam Bhattacharya, Pascaline Dupas and Shin Kanaya: Demand and Welfare Analysis in Discrete Choice Models with Social Interactions

2019-10: $\quad$ Martin Møller Andreasen, Kasper Jørgensen and Andrew Meldrum: Bond Risk Premiums at the Zero Lower Bound

2019-11: $\quad$ Martin Møller Andrasen: Explaining Bond Return Predictability in an Estimated New Keynesian Model

2019-12: $\quad$ Vanessa Berenguer-Rico, Søren Johansen and Bent Nielsen: Uniform Consistency of Marked and Weighted Empirical Distributions of Residuals

2019-13: Daniel Borup and Erik Christian Montes Schütte: In search of a job: Forecasting employment growth using Google Trends

2019-14 Kim Christensen, Charlotte Christiansen and Anders M. Posselt: The Economic Value of VIX ETPS

2019-15 Vanessa Berenguer-Rico, Søren Johansen and Bent Nielsen: Models where the Least Trimmed Squares and Least Median of Squares estimators are maximum likelihood

2019-16 Kristoffer Pons Bertelsen: Comparing Tests for Identification of Bubbles

2019-17 Dakyung Seong, Jin Seo Cho and Timo Teräsvirta: Comprehensive Testing of Linearity against the Smooth Transition Autoregressive Model

2019-18 Changli He, Jian Kang, Timo Teräsvirta and Shuhua Zhang: Long monthly temperature series and the Vector Seasonal Shifting Mean and Covariance Autoregressive model

2019-19 Changli He, Jian Kang, Timo Teräsvirta and Shuhua Zhang: Comparing long monthly Chinese and selected European temperature series using the Vector Seasonal Shifting Mean and Covariance Autoregressive model 